\title{
Paper "Differential Effect of Vitamin K and Vitamin D Supplementation on Bone Mass in Young Rats Fed Normal or Low Calcium Diet" by Iwamoto J, et al. [Yonsei Med J 2004;45(2):314-324]
}

We decided to retract the paper entitled 'Differential Effect of Vitamin K and Vitamin D Supplementation on Bone Mass in Young Rats Fed Normal or Low Calcium Diet' [Yonsei Med J 2004;45(2):314-324] by Iwamoto J, Takeda T, Ichimura S, et al. Based on information that were discovered after publication, we have recently become aware of a number of issues related to data duplication and scientific misconduct. 